\title{
¿ES POSIBLE LOGRAR UN TURISMO RURAL SOSTENIBLE EN EL CANTÓN Santa Ana de Ecuador?
}

\section{Is IT POSSIBLE TO ACHIEVE}

SUSTAINABLE RURAL TOURISM

in the Santa Ana Canton

OF ECUADOR?

\author{
Gregorio Palma Macías ${ }^{\mathrm{I}}$ \\ Marlene Cañizares Roig ${ }^{2}$
}

1 Magíster. Universidad Técnica de Manabí. Manabí, Ecuador. Correo electrónico: gpalma@utm.edu.ec

Orcid: https://orcid.org/0000-0002-1058-5159

2 Doctora en Ciencias Contables y Financieras. Universidad de La Habana. La Habana, Cuba. Correo electrónico: marlecr67@gmail.com Orcid: https://orcid.org/ 0000-0002-8433-167X

Código Jel: M41, Z32

Fecha de recepción: 19/03/2019

Fecha de aceptación: 04/04/2019

DOI: https://doi.org/10.18601/16577175.n26.05 


\section{RESUMEN}

El presente trabajo muestra avances de investigación que se desarrolla en el cantón Santa Ana de Ecuador. Se parte el diagnóstico realizado a los documentos legislados para la protección ambiental y además la identificación de actividades turísticas. Se muestran los resultados preliminares del diagnóstico y entre sus principales conclusiones señala que las actividades turísticas provocan beneficios y deterioros en el cantón, que afectan la conservación de su biodiversidad. Sin embargo, no basta con establecer políticas y regulaciones para la protección ambiental hay que monitorear los eventos que en la práctica son factores impulsores negativos del cambio en la biodiversidad y establecer mecanismos que permitan conocer los costos ambientales.

Palabras clave: turismo, rural, biodiversidad, actividades, costos ambientales.

\section{Abstract}

The present work shows advances of the research that is developed in the Santa Ana canton of Ecuador. The diagnosis made is based on the legislated documents for environmental protection and also the identification of tourist activities. The preliminary results of the diagnosis are shown and among its main conclusions it indicates that tourism activities cause benefits and deterioration in the canton, which affect the conservation of its biodiversity. However, it is not enough to establish policies and regulations for environmental protection, we must monitor the events that in practice are negative drivers of the change in biodiversity and establish mechanisms that allow us to know the environmental costs.

Keywords: Tourism, rural, biodiversity, activities, environmental costs.

\section{INTRODUCCIÓN}

Los problemas ambientales constituyen una preocupación mundial que involucran grandes desafíos interrelacionados a la pérdida de biodiversidad y el cambio climático mundial, dando lugar a una creciente sensibilización sobre la necesidad de introducir un nuevo paradigma de desarrollo, que permita calcular e incorporar aspectos sociales y económicos para la toma de decisiones.

En este escenario la biodiversidad constituye el punto de partida para transformar la matriz productiva y erradicar la pobreza. Para lograrlo es necesario realizar evaluaciones permanentes que permitan estimar la magnitud de los impactos sobre ella, así como la efectividad de las políticas públicas para prevenir, reducir y mitigar dichos impactos. Las acciones a desarrollar deben estar orientadas a la sostenibilidad de los recursos naturales. 
En este orden de ideas, se centra la atención en las actividades turísticas que directamente pueden impactar sobre los ecosistemas y la conservación de su biodiversidad. Teniendo en cuenta este aspecto, la Organización Mundial del Turismo (омт, 2017) hace mención a los cinco ámbitos claves a los que el turismo tiene que aportar una contribución esencial y duradera.

1. Crecimiento económico sostenible.

2. Inclusión social, empleo y reducción de la pobreza.

3. Uso eficiente de los recursos, protección ambiental y cambio climático.

4. Valores culturales, diversidad y patrimonio.

5. Comprensión mutua, paz y seguridad.

La población ecuatoriana cuenta con una diversidad étnica y cultural ligada a una gran variedad de destinos turísticos y numerosos sitios de gran belleza natural, lo cual dio lugar a que en las últimas décadas las comunidades comenzaran su participación en la actividad turística, desarrollando una propuesta propia: el turismo rural (Ruiz y Solís, 2007).

El turismo en espacio rural es una actividad económica en crecimiento que constituye una de las alternativas propuestas para lograr el desarrollo rural, dadas las numerosas ventajas que tiene para sus pobladores respecto a la diversificación de los ingresos, la generación de nuevos empleos, entre otros beneficios.

Esta modalidad turística se desarrolla de forma dinámica con los principios que determina la Constitución en los derechos para pueblos y nacionalidades, es decir, socialmente solidario, ambientalmente responsable, económicamente viable y culturalmente enriquecedor, por tanto, constituye una alternativa eficiente para generar fuentes de trabajo (Constitución de la República del Ecuador, 2008).

Se evidencian estudios realizados por varios autores que involucran la gestión ambiental en relación con el turismo rural, por ejemplo: Navarro y Schlüter (2010); Roux Fanny (2013); Blanco, Samper y Rivero (2014); Neudel, York (2015); Menoya, (2015); Reyes, Ortega, y Machado, (2017); Proaño y Ramírez (2017).

Según fuentes bibliográficas consultadas, en la definición sobre el turismo rural se mezclan conceptos como turismo rural, turismo cultural o ecoturismo; por ejemplo, referimos la propuesta de Navarro y Schlüter (2010), los autores caracterizan el espacio rural como aquel que presenta una estructura esencialmente agraria y artesanal, asentamiento poblacional de escasa densidad, edificación, volumetría y congestión, así como presentación paisajística en la que prevalece la naturaleza.

En este contexto Blanco, Samper y Rivero (2014) establecen que el término de turismo rural se utiliza cuando la cultura rural es un componente clave del producto ofrecido.

Como solución a la situación dada en el tratamiento conceptual fue creado el Comité de Turismo y Competitividad (стс), el mismo desde su establecimiento en el 2013, ha centrado su trabajo principalmente en evaluar el estado del conocimiento 
en torno al concepto básico de competitividad turistica y en identificar sus factores clave.

Como resultado del trabajo del CTC (2017), en la 22. ${ }^{a}$ Sesión de la Asamblea General, celebrada en Chengdu, China (11-16 de septiembre de 2017), se adoptó un conjunto de definiciones operativas de diversos tipos de turismo priorizados como turismo cultural, ecoturismo y turismo rural. Por la importancia de estos conceptos, enmarcados en su definición más actualizada, a continuación, se hacen referencia a estos:

El ecoturismo es un tipo de actividad turística basado en la naturaleza en el que la motivación esencial del visitante es observar, aprender, descubrir, experimentar y apreciar la diversidad biológica y cultural, con una actitud responsable, para proteger la integridad del ecosistema y fomentar el bienestar de la comunidad local. (p11)

El turismo cultural es un tipo de actividad turística en el que la motivación esencial del visitante es aprender, descubrir, experimentar y consumir los atractivos/productos culturales, materiales e inmateriales, de un destino turístico. Estos atractivos/productos se refieren a un conjunto de elementos materiales, intelectuales, espirituales y emocionales distintivos de una sociedad que engloba las artes y la arquitectura, el patrimonio histórico y cultural, el patrimonio gastronómico, la literatura, la música, las industrias creativas y las culturas vivas con sus formas de vida, sistemas de valores, creencias y tradiciones. (p10)

El turismo rural es un tipo de actividad turística en el que la experiencia del visitante está relacionada con un amplio espectro de productos vinculados por lo general con las actividades de naturaleza, la agricultura, las formas de vida y las culturas rurales, la pesca con caña y la visita a lugares de interés.

Las actividades de turismo rural se desarrollan en entornos no urbanos (rurales) con las siguientes características: i) baja densidad demográfica, ii) paisajes y ordenación territorial donde prevalecen la agricultura y la silvicultura, y iii) estructuras sociales y formas de vida tradicionales. (p12)

Esta definición de turismo rural esclarece la mezcla de conceptos dados en otras definiciones, por lo que se considera en función de las actividades que se desarrollan en un ambiente rural, donde se disfruta de forma general de la naturaleza, la forma de vida tradicional y las culturas rurales.

Los elementos abordados con anterioridad permiten centrar la atención en el objeto de estudio de la investigación el cantón Santa Ana, área rural donde se desarrolla actividades turísticas, entre sus competencias está la recuperación y conservación de la biodiversidad y el mantenimiento de un ambiente sostenible 


\section{Metodología}

El enfoque metodológico utilizado se desarrolló con el análisis de datos secundarios (información demográfica, informes sectoriales y locales) y primarios (entrevistas a actores representativos). El enfoque cualitativo comprendió entrevistas focalizadas a los actores representativos de distintos sectores.

En primer lugar, se parte de un análisis de los documentos que forman parte del marco regulatorio vigente para el cuidado y protección del ambiente, que garantizan que diferentes instancias de poder público ejecuten acciones conjuntas. En segundo lugar, se identifican las actividades turísticas que se desarrollan en el cantón que son la base para identificar impactos y calcular los costos de su mitigación.

\section{Marco regulatorio vigente en Ecuador para el cuidado y protección ambiental}

Para el análisis del marco regulatorio se analizaron documentos en entidades como la Asamblea Nacional del Ecuador, la Secretaría Nacional de Planificación y Desarrollo (Senplades), el Consejo Provincial de Manabí y el Municipio del cantón Santa Ana. En estos escenarios fue necesario aplicar entrevistas al personal previamente seleccionado, como resultado se identificaron los documentos que forman parte del marco regulatorio vigente para el cuidado y protección del ambiente, estos se relacionan a continuación:

Constitución de la República, Plan Nacional Toda una Vida, Ley Ambiental y Plan Nacional Ambiental, Código Orgánico Ambiental, Ley de Turismo, Ley y Reglamento del Código de las Finanzas Públicas, Agenda de Desarrollo Provincial y Plan de Desarrollo Provincial, Código orgánico de descentralización y ordenamiento territorial el plan de ordenamiento y desarrollo territorial, plan de ordenamiento territorial, Estrategia Nacional de Biodiversidad. A continuación, se describen los siguientes aspectos:

Constitución de la República del Ecuador: la Asamblea Nacional del Ecuador es el organismo rector de la aprobación de leyes en el país. En uso de sus atribuciones puso en vigor la Constitución Política Ecuatoriana, que rige desde el 2008. En el artículo 14 se reconoce el derecho de la población a vivir en un ambiente sano y ecológicamente equilibrado, que garantice la sostenibilidad y el buen vivir (sumak kawsay en lengua quechua). Se declara de interés público la preservación del ambiente, la conservación de los ecosistemas, la biodiversidad y la integridad del patrimonio genético del país, la prevención del daño ambiental y la recuperación de los espacios naturales degradados (Constitución de la República del Ecuador, 2008).

Plan Nacional de Desarrollo Toda una Vida (2017-2021): la Secretaría Nacional de Planificación y Desarrollo (Senplades), organismo rector de planificación del 
Gobierno nacional que determina las directrices de gestión pública a nivel nacional y efectúa la planificación a largo plazo, tendiente a construir una nueva forma de generación y redistribución de la riqueza para alcanzar el buen vivir. Dentro de sus competencias establece el Plan Nacional de Desarrollo Toda una Vida (20172021), este destaca doce objetivos nacionales para el desarrollo, en función de la investigación se destaca el objetivo siete que se establece en función de "garantizar los derechos de la naturaleza y promover la sostenibilidad ambiental territorial y el objetivo 8 que parte de Consolidar el sistema económico social y solidario, de forma sostenible. y global" (Senplades, 2017).

Para lograr su ejecución y monitoreo, se requiere una acción coordinada intergubernamental entre los diferentes niveles ministeriales que son decisorios y garantizan un equilibrio entre los bienes y servicios demandados y su explotación. Por ejemplo:

El Ministerio del Ambiente: institución nacional encargada de la elaboración, ejecución, monitoreo de las leyes que rigen la protección ambiental. Dentro de sus competencias está la ley de Gestión Ambiental, Código Orgánico del Ambiente y el Plan Ambiental Ecuatoriano. A continuación, se destaca sus funciones principales:

Ley de Gestión Ambiental: establece principios y directrices de política ambiental; determina las obligaciones, responsabilidades, niveles de participación de los sectores público y privado en la gestión ambiental y señala los límites permisibles, controles y sanciones en esta materia (Constitución de la República del Ecuador, 2008).

Código Orgánico del Ambiente: regula los deberes, derechos y garantías ambientales contenidos en la constitución de la República, así como los instrumentos que permiten asegurar la sostenibilidad, conservación, protección y restauración del medio ambiente (Ministerio de Ambiente de Ecuador (MAE) (2017).

El Plan Ambiental Ecuatoriano: es el instrumento técnico de gestión que promueve la conservación, protección y manejo ambiental; además, contiene los objetivos específicos, programas, acciones a desarrollar, contenidos mínimos y mecanismos de financiación, así como los procedimientos de revisión y auditoría (Ministerio de Ambiente de Ecuador (MAE) (2017).

Se considera que los diferentes planes, programas y proyectos anteriores a su ejecución deben contar con el dictamen favorable de este organismo, el cual exige que estos tengan en cuenta el respeto, cuidado, protección y mitigación de la naturaleza, garantizando la sostenibilidad de los recursos naturales.

Una vez analizados los preceptos constitucionales se logra entender que existen los elementos de juicio suficientes para que las diferentes instancias de poder público ejecuten acciones conjuntas de prevención, ejecución y evaluación del ambiente, no obstante, la problemática ambiental requiere de mecanismos específicos que garanticen su aplicación. 
Ante estos desafíos y la necesidad de costar con instrumentos y métodos que permitan su aplicación, el Programa de Naciones Unidas para el Desarrollo (PNUD) puso en marcha la iniciativa para la Financiación de la Biodiversidad (BIOFIN) como una nueva alianza mundial que procura abordar de manera integral el desafío de la financiación de las acciones orientadas hacia la biodiversidad:

El objetivo de la Iniciativa Biofin es ayudar a los gobiernos a elaborar una sólida justificación en favor de un aumento de la inversión en la conservación, el uso sostenible y la distribución equitativa de los beneficios de los ecosistemas y la biodiversidad. El Manual de Biofin es una herramienta que ayuda a cuantificar el déficit de financiación de la biodiversidad a nivel nacional, con el fin de mejorar la eficacia en función del costo mediante la incorporación de la biodiversidad en el desarrollo nacional y la planificación sectorial, así como de elaborar estrategias integrales de movilización de recursos a nivel nacional. (PNUD, 2014, p. 9)

Ecuador forma parte de este proyecto Biofin en la vanguardia mundial del respeto a la naturaleza y a los pueblos. Según se plasma en el texto constitucional, un modelo sustentable de desarrollo, ambientalmente equilibrado y respetuoso de la diversidad cultural, que conserve la biodiversidad y la capacidad de regeneración natural de los ecosistemas y asegure la satisfacción de las necesidades de las generaciones presentes y futuras (Constitución de la República del Ecuador, 2008).

La biodiversidad es considerada un recurso estratégico para el desarrollo, desafío que el Gobierno nacional emprendió con un nuevo proceso de planificación para la gestión de la biodiversidad en el periodo 2015-2030. Fue así que se elaboró la Estrategia Nacional de Biodiversidad 2015-2030, que toma como principales puntos de referencia el Plan Nacional para el Buen Vivir 2013-2017 y el Plan Estratégico para la Diversidad Biológica 2011-2020. Forman parte de este plan veinte metas agrupadas en cinco objetivos estratégicos, que se conocen como Metas de Aichi, dichas metas están alineadas con los objetivos de desarrollo sostenible y buscan principalmente: mitigar las causas por las cuales se está perdiendo la biodiversidad desde los ámbitos sociales y políticos; reducir las presiones que enfrenta y promover la utilización sostenible de los recursos naturales; mejorar la situación de la biodiversidad a través del cuidado de sus ecosistemas; optimizar los beneficios que la naturaleza nos brinda (aire limpio, agua dulce, alimentos) (Ministerio del Ambiente de Ecuador, 2016).

El art. 395 de la Constitución establece cuatro principios ambientales que constituyen los pilares de la Estrategia Nacional de Biodiversidad:

1. El Estado garantizará un modelo sustentable de desarrollo, ambientalmente equilibrado y respetuoso de la diversidad cultural que conserve la biodiversidad y la capacidad de regeneración natural de los ecosistemas y asegure la satisfacción de las necesidades de las generaciones presentes y futuras. 
2. Las políticas de gestión ambiental se aplicarán de manera transversal y serán de obligatorio cumplimiento por parte del Estado en todos sus niveles y por todas las personas naturales o jurídicas en el territorio nacional.

3. El Estado garantizará la participación activa y permanente de las personas, comunidades, pueblos y nacionalidades afectadas, en la planificación, ejecución y control de toda actividad que genere impactos ambientales.

4. En caso de duda sobre el alcance de las disposiciones legales en materia ambiental, estas se aplicarán en el sentido más favorable a la protección de la naturaleza.

Otras acciones se reflejan en los resultados alcanzados por la integración con varios convenios internacionales para la gestión ambiental, como los siguientes:

- Convenio sobre la diversidad biológica.

- Convenio marco de las Naciones Unidas sobre cambio climático.

- Convenio de lucha contra la desertificación y la sequía.

En consecuencia, con todos estos documentos rectores que hoy están vigentes se considera que constituye un importante reto para el Estado ecuatoriano, asumir las obligaciones derivadas de estos instrumentos, garantizando la articulación de los distintos actores e intereses del sector público, privado y comunitario.

Para verificar la ejecución práctica de las disposiciones legales, se realizó una triangulación de fuentes, centrando la atención en dos nuevos escenarios, Manabí y el cantón Santa Ana.

Manabí dentro de su estructura políticoadministrativa tiene el Gobierno provincial, 22 gobiernos autónomos descentralizados y sus 77 gobiernos parroquiales, cada uno con sus competencias específicas.

Para la ejecución, monitoreo y evaluación de las leyes ambientales la provincia de Manabí cuenta con el Gobierno provincial, este elabora el Plan de Desarrollo y Ordenamiento Territorial de Manabí y la Agenda Provincial 2011-2020. En esta se enmarcan las políticas que impulsan el proceso de construcción del desarrollo social y económico territorial para ejecutar planes, programas y proyectos que garanticen el progreso, la calidad de vida y la riqueza sustentable de la población, en especial, lo relacionado a la gestión ambiental por los variados ecosistemas con multiplicidad de especies vegetales y animales que permitan mitigar el deterioro que afrontan actualmente.

En dicha provincia se concentra la atención en el cantón Santa Ana, donde el Gobierno autónomo descentralizado de este ha emitido ordenanzas relacionadas con la recolección, disposición y tratamiento de los desechos sólidos, permisos y controles ambientales en las zonas urbanas y rurales, control de ruido, contaminación ambiental y, en concordancia, con la constitución vigente, el Plan Nacional Desarrollo Toda una Vida y el Gobierno Provincial para lograr un equilibrio entre los recursos naturales existentes y una adecuada explotación racional de sus recursos. 
Como resultado de esta triangulación entre los documentos rectores vigentes y su aplicación práctica se arriban a las siguientes conclusiones:

- Es evidente que existe un marco regulatorio para el cuidado ambiental que se manifiesta desde el contexto nacional, provincial y local, este se articula con los planes, programas y proyectos enmarcados en la planificación gubernamental y en todas las esferas del poder público.

- En la práctica existe carencia de monitoreo y evaluación de eventos ambientales que inciden en su aplicación desde los distintos escenarios, lo que se ve reflejado en los esfuerzos individuales de cada organización y entidades para lograr que los resultados sean adecuados.

Los elementos abordados en el marco regulatorio para el cuidado y protección del ambiente en Ecuador conducen a analizar su aplicación en el contexto local, para ello se tomó como referencia el cantón Santa Ana, que se considera un asentamiento donde el turismo comunitario rural puede ser un modelo de gestión adecuado para esta zona.

\section{Las actividades turísticas del cantón Santa Ana}

El cantón Santa Ana geográficamente se encuentra ubicado en el centro sur de la Provincia de Manabí, en un hermoso valle rodeado por los cerros: Peminche y Bonce al noroeste, el cerro Mate al sur, las Guaijas y las montañas del Sasay al oeste; limita con varios cantones: al norte con Portoviejo, al sur con Olmedo y 24 de mayo, al este con Pichincha y al oeste con 24 de mayo, Jipijapa y Portoviejo. Está dividido en seis parroquias, dos de ellas urbanas que son Santa Ana de Vuelta Larga y Lodana, y cuatro rurales: Ayacucho, Honorato Vásquez, La Unión y San Pablo de Pueblo Nuevo. Su densidad poblacional es de 43.67 habitantes por kilómetro cuadrado y su población alcanza la cifra de 47385 , que representa el $3.4 \%$ del total de la provincia de Manabí.

El $52.94 \%$ de su población reside en el área rural y el $47.06 \%$ en el sector urbano y se caracteriza por ser una población joven, ya que el $51 \%$ son menores de 24 años, según la pirámide de población por edades y sexo insertos en los resultados del censo (INEC, 2017).

Sus ríos y montañas forman un corredor turístico bañado por las aguas del río Portoviejo, su caudal forma la represa Poza Honda, esta es utilizada para el consumo humano, riego y animales y tiene una capacidad de cien millones de metros cúbicos, que abastece la población de siete cantones

Santa Ana no solo cuenta con amplias áreas rurales nutridas de recursos naturales también representa cultura, folklore, tradiciones orales, como amorfinos, cuentos y leyendas, lo que constituye un patrimonio intangible que precisa ser conservado para no perder esa identidad. Además, se considera uno de sus principales objetivos 
mejorar el nivel de vida de sus habitantes en las comunidades, así como también contribuir a la preservación de su territorio, mediante prácticas de turismo sostenible.

Para la caracterización del cantón, se realizaron visitas de campo que garantizaron la observación del área objeto de estudio, se consultaron páginas web del Instituto Nacional de Estadística y Censos (INEC) que brindan datos geográficos y estadísticos y se aplicaron entrevistas y encuestas.

Las características naturales del cantón Santa Ana permiten realizar actividades en un ambiente rural, donde se disfruta de forma general de la naturaleza, de la vida tradicional y las culturas; considerado, según los fundamentos teóricos, un turismo rural.

\section{Resultados y Discusión}

El desarrollo del turismo rural puede impactar sobre la conservación de la biodiversidad, sino se ejecutan acciones que tributen a su sostenibilidad, entonces, ¿es posible lograr un turismo rural sostenible en el cantón Santa Ana?

Para lograr prácticas sostenibles, se hace necesario identificar las actividades turísticas que se realizan en el cantón. Se aplicaron encuestas a la comunidad y a personal técnico del área seleccionada, como resultado del total de encuestas aplicadas el $77.50 \%$ identifican las actividades turísticas que se desarrollan en el cantón.

Una vez identificadas las actividades se presentaron los resultados al grupo de expertos que participan en la investigación, como resultado fueron seleccionadas las actividades que se muestran en el gráfico 1 .

Gráficol. Actividades turísticas que se realizan en el cantón Santa Ana

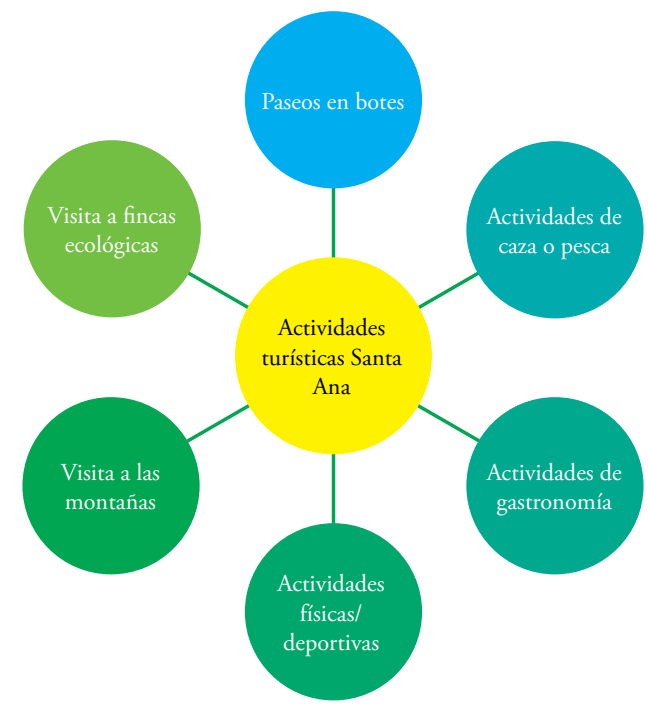

Fuente: elaboración propia. 
Las actividades turísticas provocan beneficios y deterioros en el cantón que afectan la conservación de su biodiversidad. Dentro de los principales beneficios se destacan: nuevas fuentes de empleo, disminución de la pobreza, alimentación y hospedaje, nuevas fuentes de ingresos para las localidades.

Por otro lado, están los deterioros provocados como son: contaminación del agua, aumento de los asentamientos de la población en áreas no permitidas, agotamiento de la fuente natural de belleza escénica, erosión del suelo, pérdida de la flora y fauna.

Significa que para lograr un turismo sostenible hay que articular acciones económicas, sociales y ambientales como pilares de la sostenibilidad. Según el diagnóstico realizado en el marco regulatorio, se identifica que se cuenta con políticas y leyes que rigen la protección ambiental; sin embargo, la dificultad se presenta en la carencia de mecanismos que involucran el cálculo de los costos y su importancia para la toma de decisiones en la sostenibilidad ambiental.

Se considera que lograr internalizar aspectos económicos es un eje central en el logro de la sostenibilidad del cantón porque permitirá no solo realizar acciones de gestión ambiental, sino contar con mecanismos económicos que posibiliten calcular el costo de mitigar los impactos provocados a la biodiversidad y el logro de su conservación. De esta manera, las actividades turísticas del cantón Santa Ana estarían aportando al crecimiento económico sostenible; a la inclusión social, empleo y reducción de la pobreza; al uso eficiente de los recursos y a la protección ambiental.

¿Se cuenta con mecanismos para ejecutar estas acciones?

En este análisis se ha podido determinar que en el Ecuador no ha prosperado la contabilidad ambiental, porque no existen normas contables que obliguen a las empresas a registrar y revelar información de las operaciones económicas de los hechos ambientales que provocan.

En la actualidad se trabaja para enfrentar esta problemática, se cuenta con metodologías que abordan el cálculo del costo integrado a la gestión ambiental como se refiere en el Manual de BIofin, que presenta un enfoque más simplificado para el cálculo de los costos, al relacionar las Metas de Aichi para la diversidad biológica con los objetivos estratégicos y las acciones definidas en el Manual de BIOFIN, esta relación se muestra en el gráfico 2 .

Se trata de mostrar avances que forman parte de la aplicación del manual Biofin, tomando como ejemplo la estrategia de restauración, acción que debe ejecutarse producto a los impactos provocados por las actividades turísticas que fueron identificadas anteriormente y que permitirá, en el trascurso de la investigación, el cálculo de los costos ambientales. 
Gráfico 2. Calcular los costos ambientales integrando la gestión ambiental con las acciones definidas en el Manual de BIOFIN

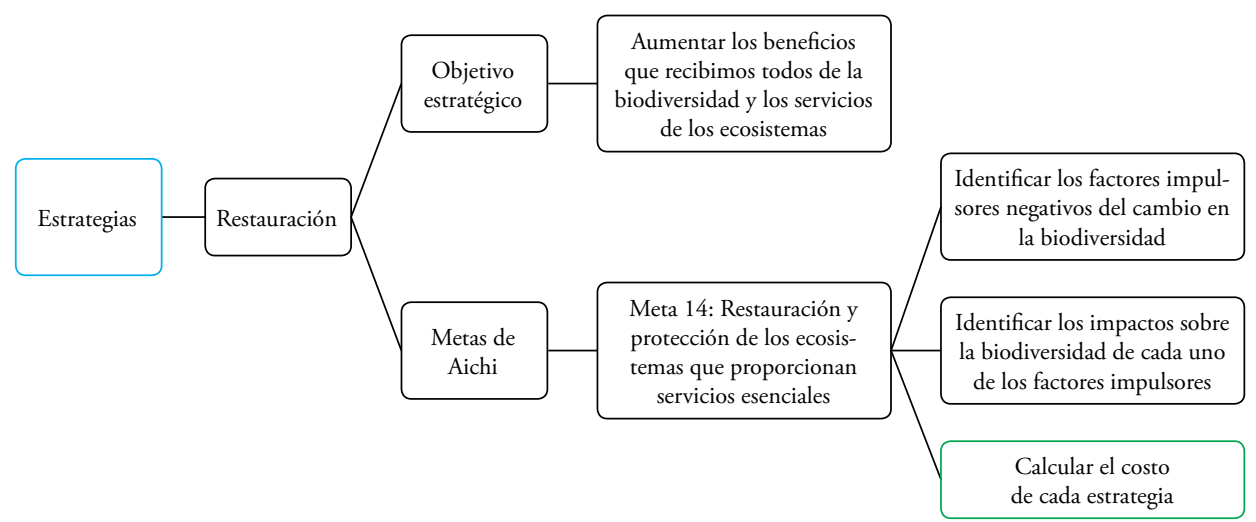

Fuente: elaboración propia a partir del Manual de BIOFIN.

\section{Conclusiones}

Se considera que las fuentes consultadas en la investigación (las regulaciones vigentes para la protección ambiental, las metas y los objetivos estratégicos) pueden articularse entre sí, ya que todas tiene un objetivo en común: la protección de la biodiversidad como estrategia de desarrollo.

El cantón Santa Ana de Ecuador, se caracteriza por su belleza natural que permite realizar actividades en un ambiente rural, donde se disfruta de la naturaleza, la forma de vida tradicional y las culturas, escenario que facilita el turismo rural.

Las actividades turísticas provocan beneficios y deterioros en el cantón que afectan la conservación de su biodiversidad, pero no basta con establecer políticas y regulaciones para la protección ambiental, hay que monitorear los eventos que en la práctica son factores impulsores negativos del cambio en la biodiversidad y establecer mecanismos que permitan conocer los costos de prácticas actuales y el costo de monitorear y ejecutar acciones futuras

Para lograr un turismo rural sostenible en el cantón es necesario establecer mecanismos de control que permitan registrar, calcular y evaluar los costos de las acciones de los diferentes agentes agresores sobre los ecosistemas, logrando la internalización de aspectos ambientales, económicos y sociales como pilares de la sostenibilidad.

\section{REFERENCIAS BIBLIOGRÁFICAS}

Asamblea Constituyente (2008). Constitución de la República de Ecuador. MontecristiEcuador. 
Blanco, M., Samper, M. y Riveros, H. (2014). Guía para la elaboración del plan de desarrollo turistico de territorios rurales. Convenio de colaboración entre IICA Costa Rica y el Programa de Desarrollo Agroindustrial Rural (Prodar). Costa Rica: IICA-Prodar.

Comité de Turismo y Competitividad (стC) (2017). Definiciones de turismo. Disponible en http://cf.cdn.unwto.org/sites/all/files/pdf/definicionesde turismo de la omt.pdf. Consultado enero 2019

Instituto Nacional de Estadística y Censos (INEC) (2017). Dirección de Estudios Laborales y Económicos. Disponible en http://www.ecuadorencifras.gob.ec/documentosLibros/ Demografia/documentofinal1.pdf. Consultado junio 2018

Menoya, S. (2015). Modelo de gestión del turismo desde el gobierno local para municipios con vocación turistica basado en el enfoque de cadena de valor. Tesis presentada en opción al grado científico de doctor en Ciencias Económicas, Universidad de Pinar del Río, Cuba.

Ministerio de Ambiente de Ecuador (mae) (2017). Código Orgánico del Ambiente. Ley Ambiental. Ministerio de Ambiente de Ecuador, Quito-Ecuador. Disponible en ww.lacamara.org/website/wp-content/uploads/2017/03/IPE-269-Código-Orgánicodel-Ambiente.pdf

Ministerio de Ambiente de Ecuador (MAE) (2017). Ley Ambiental. Ministerio de Ambiente de Ecuador, Quito-Ecuador. Disponible en http://www.ambiente.gob.ec/wpcontent/ uploads/downloads/2012/09/LEY-DE-GESTION-AMBIENTAL.pdf

Ministerio de Turismo (mintur) (2015). Resultados del 2015, año de la Calidad Turística en Ecuador Disponible en www.turismo.gob.ec

Ministerio del Ambiente del Ecuador (2016). Estrategia Nacional de Biodiversidad 20152030, primera edición, noviembre de 2016, Quito-Ecuador. Consultado diciembre 2018 Disponiblehttp://www.ec.undp.org/content/dam/ecuador/docs/documentos\%20 proyectos $\% 20$ ambiente/Estrategia $\% 20$ Nacional\%20de $\% 20$ Biodiversidad $\% 20$ 2015-2030\%20-\%20CALIDAD\%20wEB.pdf

Navarro, F. y Schlüter, R. (2010). El turismo en los pueblos rurales de Argentina: ¿es la gastronomía una opción de desarrollo? Estudios y perspectivas en turismo, 19(6), 909-929.

Neudel, Y. (2015). La experiencia del turismo comunitario en Yunguilla, Ecuador y su impacto sociocultural en la comunidad. Letras Verdes. Revista Latinoamericana de Estudios Socioambientales, 18(3), 48-70.

Organización Mundial del turismo (омт) (2017). Informe sobre el Año Internacional del Turismo Sostenible para el Desarrollo, 2017. Organismo especializado de las Naciones Unidas. Disponible en www.tourism4development2017.org/wpcontent/ uploads/2017/05/12522_iy2017-discussion-paper_executive-summary

Proaño Ponce, W. P. y Ramírez Pérez, J. F. (2017). Modelo de desarrollo turístico sostenible para cantones costeros: herramienta para actores locales de Manabí, Ecuador. Revista Posgrado y Sociedad. Sistema de Estudios de Posgrado, 15(2),65-78.

Programa de Naciones Unidas Para el Desarrollo (pNUD) (2014). Manual de BIofin. Una herramienta para movilizar recursos financieros para la biodiversidad y el desarrollo Programa de Naciones Unidas para el Desarrollo. Nueva York. 
Reyes, M., Ortega, Á. y Machado, E. (2017). Modelo para la gestión integrada del turismo comunitario en Ecuador, caso de estudio Pastaza. REVESCO Revista de Estudios Cooperativos. Recuperado de https://www.researchgate.net/publication/311515318

Roux, F. (2013). Turismo comunitario ecuatoriano, conservación ambiental y defensa de los territorios. Quito: FEPTCE.

Ruiz, E. y Solís, D. (2007). Turismo comunitario en Ecuador: desarrollo y sostenibilidad social. Quito: Ecuador.

Senplades (2017). Plan Nacional de Desarrollo/Plan Nacional para el Buen Vivir 20172020. Quito, Ecuador. 